\title{
Comparing the Effects of Ginger and Glibenclamide on Dihydroxybenzoic Metabolites Produced in Stz-Induced Diabetic Rats
}

\author{
Ramesh Ahmadi ${ }^{1,{ }^{*}}$, Saeede Pishghadam ${ }^{2}$, Fatemeh Mollaamine ${ }^{3}$, Mohammad Reza Zand \\ Monfared $^{3}$ \\ ${ }_{2}^{1}$ Department of Physiology, Islamic Azad University, Qom, IR Iran \\ ${ }_{3}^{2}$ Islamic Azad university, Qom, IR Iran \\ 3 Department of Chemistry, Islamic Azad university, Qom, IR Iran \\ *Corresponding author: Ramesh Ahmadi, Islamic Azad university of Qom, 15 Khordad St, Qom, IR Iran. Tel:+98-2537780001, Fax:+98-2137770001, E-mail: ramahmd@yahoo.com.
}

Received: March 16, 2013; Revised: April 30, 2013; Accepted: May 27, 2013

\begin{abstract}
Background:The aim of the present study was to investigate the effect of ginger and glibenclamide on oxidative stress markers. Oxidative stress is caused by an unbalance between a relative overload of oxidants and depletion of antioxidants, as implicated in the pathogenesis of several chronic diseases, including atherosclerosis and diabetes mellitus. Regarding the role of oxidative stress in the pathogenesis of diabetes mellitus, we investigated the effect of ginger and glibenclamide in diabetic rats induced bystreptozocin(STZ).

Objectives: This study assessed the effects of ginger and glibenclamide on dihydroxybenzoic acid metabolites in diabetic rats.

Materials and Methods: In this study 30 Wistar strain male rats were divided into five groups: Group 1: Normal control receiving normal saline (0.9 0/0), Group2: control DMSO (Dimethyl sulfoxide)(as solvent of glibenclamide), Group 3: Diabetic control receiving Streptozocin (STZ ) (50 mg/kg), Group 4: diabetic+ Ginger Extract: this group received ginger ethanolic extract ( $200 \mathrm{mg} / \mathrm{kg}$ ) via IP (Intraperitoneally) injection for 30 days, and Group 5 diabetic rats received glibenclamide $(0.5 \mathrm{~m} / \mathrm{kg})$. Production of hydroxyl radicals was examined in the diabetic rats induced by streptozocin. Hydroxyl radicals were generated in plasma of the hyperglycemic rats, and were quantitatively assayed by trapping hydroxyl radicals with salicylic acid so as to produce 2,3-and 2,5-dihydroxybenzoic acid.

Results: Production of hydroxyl radicals increased; therefore, by using salicylic acid, hydroxyl radicals were trapped and 2,3dihydroxybenzoic acid and 2,5dihydroxybenzoic acid metabolites were formed then measured by HPLC and spectrophotometer. Rats receiving ginger extract and glibenclamide showed decreased level of metabolites compared to the diabetic controls $(\mathrm{P}<0 / 001)$. This means that antioxidants act as scavenger of free radicals.

Conclusions: Comparative effect of ginger and glibenclamide also showed that glibenclamide has antioxidant effect as a scavenger of free radical, but ginger is more capable of eliminating them.
\end{abstract}

Keywords: Free Radical; STZ-Induced Diabetic Rats; Ginger; Glibenclamide

\section{Background}

Oxidative stress is caused by an unbalance between a relative overload of oxidants and a depletion of antioxidants (1-4). In the research field of free radicals in biological samples it is still a major problem to determine the amount of free radical damage. One of the most aggressive radicals is the hydroxyl radical (5-7). Oxidative stress is currently suggested as the mechanism underlying diabetes and diabetic complications (8).

Hyperglycemia, several other factors like hyperlipidemia and enhanced oxidative stress play a major role in diabetic pathogenesis. The disease is progressive and is associated with high risk of complications (9). During diabetes, persistent hyperglycemia causes increased production of free radicals, especially reactive oxygen species (ROS), for all tissues from glucose auto-oxidation and protein glycosylation. Various mechanisms have been suggested to contribute to the formation of these reactive oxygen-free radicals. Glucose oxidation is believed to be the main source of free radicals (10). Pancreatic beta cells are particularly sensitive to be damaged via reactive oxygen species (ROS), whether generated by the complex pro inflammatory environment of an autoimmune cellular infiltrate (insulitis), or by the free radical generating toxin alloxan(11, 12). Oxidative stress is thought to be a major risk factor in the onset and progression of diabetes. Many of the common risk factors, such as obesity, increased age, and unhealthy eating habits, all contribute to an oxidative environment (13). Ginger (Zingiber officinale) is widely consumed as spice for the flavoring of foods. Ginger is reported to have several beneficial pharmacological effects (hypoglycemic, insulin tropic,

Implication for health policy/practice/research/medical education:

This paper emphasize on more need for using of antioxidant which protect the key cell components by neutralizing the damaging effects of free radicals Copyright @ 2013, Research Institute For Endocrine Sciences and Iran Endocrine Society; Published by Kowsar. This is an Open Access article distributed under the terms of the Creative Commons Attribution License (http://creativecommons.org/licenses/by/3.0), which permits unrestricted use, distribution, and reproduction in any medium, provided the original work is properly cited. 
and hypolipidemic) on health in humans (10) and in experimental animals $(14,15)$. It has been reported that ginger or its extracts possesses some pharmacological activities including anti emesis (16), analgesic effect (17), antitumor (18) and antioxidant (19). The active ingredients in ginger root include volatile oils and pungentphenol compounds known as gingerols, sesquiterpenoids, and shogaols (20). Zancan et al. mentioned that all major active ingredients of $Z$. officinale roots and leaves such as zingerone, gingerdiol, zingiberene, gingerols and shogaols have antioxidant activity (21). Since the effect of ginger and glibenclamide have not been compared for their antioxidant action on diabetic rats ,thus, the present study aimed to investigate comparative effect of ginger and glibenclamide on oxidative damage in streptozocin induced diabetic rats.

\section{Objectives}

The aim of the study was to compare the effects of ginger and glibenclamide on dihydroxybenzoic acid metabolites produced in diabetic rats.

\section{Materials and Methods}

\subsection{Animals}

Wistar male rats weighing $250 \pm 25 \mathrm{~g}$ were purchased from Karaj Pastor Institute. Rats were housed in clean plastic cages of six rats per each. Ambient temperature of animal room was $\left(27 \pm 2^{\circ} \mathrm{C}\right)$ with a 12 hours dark and light cycle (22). Rats were given standard pellets diet and water (22) throughout the experimental period.

\subsection{Chemicals}

STZ was obtained from Sigma chemicals (The USA).

\subsubsection{Induction of Diabetes}

The animals were fasted overnight and diabetes was induced by a single in traperitoneal injection of a freshly prepared solution of streptozocin (STZ) dissolved in physiological saline ( $50 \mathrm{mg} / \mathrm{kg}$ body weight).The animals were considered as diabetic, if their blood glucose values were above $250 \mathrm{mg} / \mathrm{dL}$ on the third day after the STZ injection. The blood glucose was measured by glucometer.

\subsection{Ginger Ethanolic Extract Preparation}

Two kilograms of fresh rhizomes of ginger was purchased from a local market, peeled, washed, dried, and pulverized with a blender to a fine powder, and extracted in Soxhlet extractor with $95 \%$ ethanol for 24 hours,and the extraction was continued, filtered and the filtrate was concentrated to dryness under reduced pressure in arotary evaporator and then the extract was transported to Desiccator. The result in get hanolic extract was air-dried; finally resulting 80 grams of dark brown, gelatinous extract of gingerdried rhizomes. Without any further purification, the crude ethanolic extract was used for the experiments. Dose equivalent to $200 \mathrm{mg}$ of the crude extract per kg body weight, was calculated (22).

\subsection{Grouping of Animals}

The rats were divided into five groups, six rats in each group and treated as follows: Including, normal control group (NC) which received normal saline (0.9\%), and fed with normal diet, normal control which received dose of $0 / 5 \mathrm{mg} / \mathrm{kg}$ of DMSO (Dimethyl sulfoxide) (as a solvent of glibenclamide) and diabetic control group which was given streptozocin (STZ) intra peritoneally with a single dose of $50 \mathrm{mg} / \mathrm{kg}$, and diabetes plus dose of $200 \mathrm{mg} /$ $\mathrm{kg}$ ginger extract $(\mathrm{D}+\mathrm{Gli})$ and diabetics rats which were treated with a dose of $1 \mathrm{mg} / \mathrm{kg}$ glibenclamide for 30 days. Salicylic acid ( $22 \mathrm{mg} / \mathrm{kg}$ body weight) was injected to each rat. One hour after salicylic acid injection, rats were killed and blood was drawn from the left ventricle of heart and collected in a heparin-containing tube. By centrifuging at $2500(\mathrm{rpm})$ for $20 \mathrm{~min}$, plasma $(1000 \lambda)$ was prepared for the measurement of hydroxyl radicals. Plasma was mixed with distilled water (10 times).The supernatant was filtered through a $0 / 45,0 / 22(\mu \mathrm{m})$ filter and assayed by HPLC.

\subsection{Determination of 2,3-and 2,5-DHBA by HPLC (23)}

The filtered sample ( 0.45 and $0.22 \mu \mathrm{m})$ was applied on to an HPLC apparatus, composed of a V7603 HPLC pump from, and a V7604 UV detector from KNAVER equipped with a flow cell operating at 280 and $305 \mathrm{~nm}$. The analyses were performed at room temperatures using aRP18 $250 \times 4.6 \mathrm{~mm}$ SS EXSIL ODS $5 \mu \mathrm{m}$ and a flow rate mobile phase (Me OH:10/01 aqueous HOAC (80:20) 1mL/min.

\subsection{Statistical Analysis}

Analysis of one way ANOVA was used. Data were analyzed using the SPSS (Version SPSS15). Statistical significance was set at $\mathrm{P}<0.05$.

\section{Results}

\subsection{Effect of Ginger and Glibenclamide on the Blood Glucose Levels and Body Weight Changes}

The STZ-induced diabetic rats showed significant increase of blood glucose levels in comparison to normal control rats, which further increased during the experimental period. Intra peritoneally injection of ginger showed drastic increased levels in blood glucose in diabetic group, and body weight was lower than the control group (Table 1). 
Ahmadi R et al.

Table 1. Blood Glucose Levels and Body Weight Changes in STZ-induced Rats Followed by Ginger and Glibenclamide Treatment.

\begin{tabular}{lllll}
\hline Group & \multicolumn{2}{c}{ Blood Glucose, $\mathbf{~ n m o l} / \mathbf{m L}^{\mathrm{a}}$} & & ${\text { Body Weight, } \mathbf{g}^{\mathrm{a}}}^{\text {0 }}$ \\
& 0 th Day & 30 th Day & 0 th Day & 30 th Day \\
\hline Group 1 (NC) & $78 \pm 2.2$ & $98 \pm 1.8$ & $212 \pm 8.66$ & $239 \pm 25$ \\
\hline Group 2 (DMSO) & $100 \pm 1.1$ & $105 \pm 2.3$ & $204 \pm 20$ & $245 \pm 19.1$ \\
\hline Group 3 (DC) & $463 \pm 2.2^{\mathrm{b}}$ & $501 \pm 2.14^{\mathrm{b}}$ & $181 \pm 15.94^{\mathrm{b}}$ & $211 \pm 10.5$ \\
\hline Group 4 (Gt) & $455 \pm 3.25^{\mathrm{C}}$ & $260 \pm 2.2$ & $211 \pm 11.3$ & $212 \pm 47^{\mathrm{C}}$ \\
\hline Group 5 (Gilt) & $377 \pm 3.3^{\mathrm{C}}$ & $202 \pm 2.2^{\mathrm{C}}$ & $193 \pm 19.7$ & $216 \pm 32^{\mathrm{C}}$ \\
\hline
\end{tabular}

$\mathrm{a}(\mathrm{P}<0.01)$

$\mathrm{b}$ All the values are mean $\pm \mathrm{SD}$ of five individual observations.

${ }^{\mathrm{C}}$ Values are significant compared to the normal control $(\mathrm{P}<0.001)$ and diabetic control

\subsection{Comparing the Production of 2, 3DHBA in Val- ue Between the Groups}

Figure 1 Represents the Activity of Ginger Antioxidant and Decreased Level of2, 3-DHBA Metabolite in Rats Induced Diabetes Treated by Ginger Compared to glibenclamide $(\mathrm{P}<0.01)$ and Diabetic Groups $(\mathrm{P}<0.001)$.

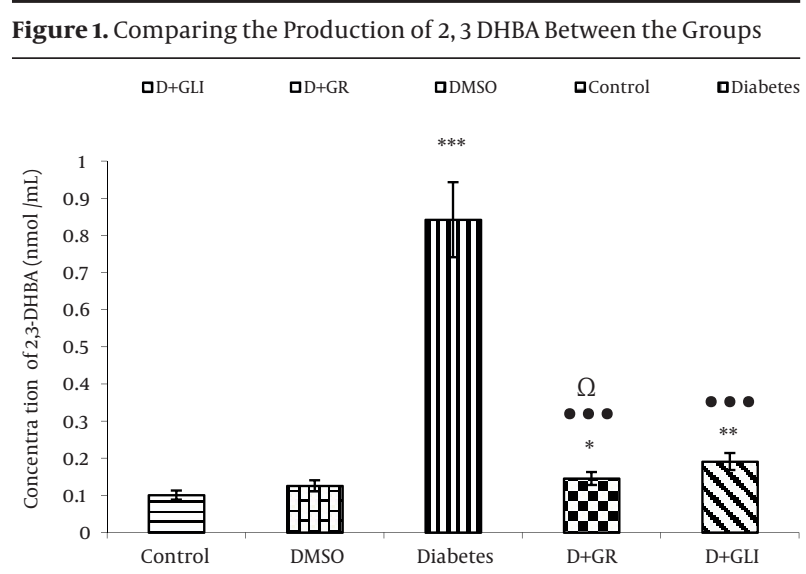

* shows the comparison between the control group and all other groups. - shows the comparison between the treatment groups with diabetic control. $\Omega$ shows the comparison between the treatment groups.

\subsection{Comparing the Production of 2, 5DHBA Be- tween the Groups}

Figure 2 also shows the activity of antioxidant of ginger in low production of 2, 5 DHBA compared to glibenclamide $(\mathrm{P}<0.01)$ and diabetic group $(\mathrm{P}<0.001)$.

\section{Discussion}

Our experimental findings showed that diabetic rats which received ginger extract had increased levels of 2 , 3-DHBA and 2, 5-DHBA metabolites $(\mathrm{P}<0.01)$. The antioxidant compounds and other pharmacological compounds of ginger may inhibit the production of free radicals. After 30 days, supplementation of ethanolic extract of ginger to diabetic rats, resulted in more significant diminution of 2,3-DHBA and 2,5-DHBA levels than diabetic rats administrated glibenclamide.

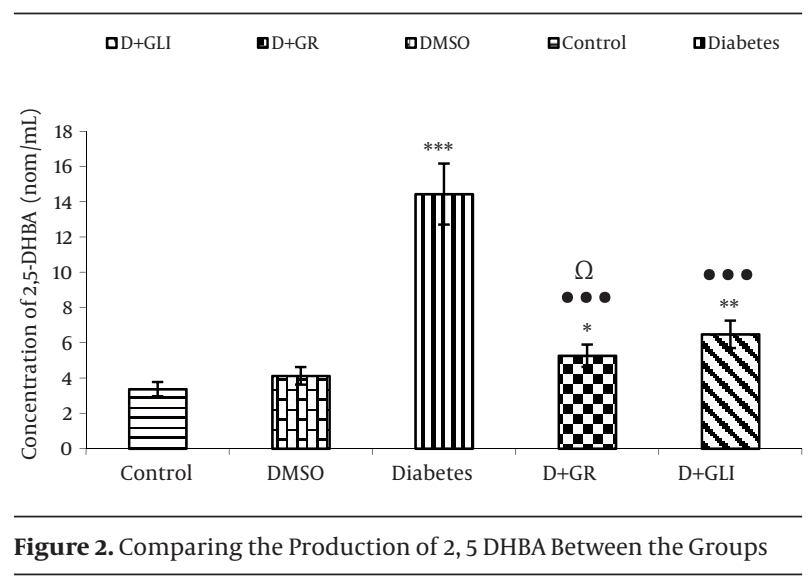

In STZ-induced diabetic rats hydroxyl radicals are increased significantly in plasma and it is detectable by increased levels of 2,3-DHBA and 2,5-DHBA. The present study clearly demonstrated that hydroxyl radical formation was markedly enhanced in plasma by induction of hyperglycemia with STZ. Diabetes is associated with a higher oxidative stress. ROS induced damage to the insulin producing pancreatic beta-cells induces diabetes (24). Salicylate has good properties to actas an in vivo marker for oxidative determine salicylate and its free radical products stress (25). Hydroxyl free radical may react with salicylate to form 2.3-dihydroxybenzoic acid(2.3DHBA) A and 2.5-dihydroxybenzoic acid (2.5DHBA) (23). Intermittent increases in ambient glucose concentrations are associated with increased production of reactive oxygenspecies (ROS) in vivo as well as in cell cultures(26-28). Diabetes mellitus affects approximately 100 million people (29), and its prevalence has been increasing (30). Oxidative stress can be defined as a state of imbalance toward the factors that generate reactive oxygen radicals (e.g., superoxide or hydroxyl radicals) (24). Under conditions of oxidative stress, free radicals that are not reduced or removed from the cellular environment can cause damage 
to all cellular macromolecules including nucleic acids, lipids, and proteins (31). Streptozocin injection resulted in diabetes mellitus, which may be due to the destruction of beta cells of Islets of Langerhans as proposed by others (32). Diabetes arises from irreversible destruction of pancreatic beta cells, causing degranulation and reduction of insulin secretion (33). During diabetes, persistent hyperglycemia causes increased production of free radicals, especially reactive oxygen species (ROS) (34). Glibenclamide eliminates reactive oxygen species (ROS)(35, 36) comparable with glibenclamide. Ginger is a standard hypoglycemic medication. Hydroxyl radical $(\mathrm{OH})$ is the most reactive oxygen species (ROS), which can act with most organic molecules added to unsaturated bond, hydrogen abstraction, or electron transfer (its reduction potential 1.9 V) (37) Salicylate has good properties to act as an in vivo marker for oxidative stress (24).

\section{Acknowledgements}

The authors wish to thank the participants of the study. We have special thanks physiology and chemistry department.

\section{Authors' Contribution}

All authors have similar contributions in writing proposal, sampling, data analysis and manuscript preparation.

\section{Financial Disclosure}

There is no Financial Disclosure.

\section{Funding/Support}

There is no Funding/Support.

\section{References}

1. Al Ghouleh I, Khoo NK, Knaus UG, Griendling KK, Touyz RM, Thannickal VJ, et al. Oxidases and peroxidases in cardiovascular and lung disease: new concepts in reactive oxygen species signaling. Free Radic Biol Med. 2011;51(7):1271-88.

2. Del Vecchio L, Locatelli F, Carini M. What we know about oxidative stress in patients with chronic kidney disease on dialysis-clinical effects, potential treatment, and prevention. Semin Dial. 2011;24(1):56-64.

3. Droge W. Free radicals in the physiological control of cell function. Physiol Rev. 2002;82(1):47-95.

4. Rains JL, Jain SK. Oxidative stress, insulin signaling, and diabetes. Free Radic Biol Med. 2011;50(5):567-75.

5. Burk Raymond F, Ludden Thomas M. Exhaled alkanes as indices of in vivo lipid peroxidation. Biochemical Pharmacology. 1989;38(7):1029-1032.

6. Wade Chris R, van Rij AndréM. In vivo lipid peroxidation in man as measured by the respiratory excretion of ethane, pentane, and other low-molecular-weight hydrocarbons. Anal Biochem. 1985;150(1):1-7.

7. Wendel Albrecht. Measurement of in vivo lipid peroxidation and toxicological significance. Free Radical Biol Med.1987;3(5):355-358.

8. Moussa SA. Oxidative stress in diabetes mellitus. Romanian J Biophys. 2008;18(3):225-236

9. Dewanjee Saikat, Bose Sekhar K, Sahu Ranabir, Mandal Subhash C. Antidiabetic effect of matured fruits of Diospyros peregrina in alloxan-induced diabetic rats. Int J Green Pharm. 2008;2(2):95.

10. Evans JL. Oxidative Stress and Stress-Activated Signaling Pathways: A Unifying Hypothesis of Type 2 Diabetes. Endocrine Reviews. 2002;23(5):599-622.

11. Takasu N, Asawa T, Komiya I, Nagasawa Y, Yamada T. Alloxan-induced DNA strand breaks in pancreatic islets. Evidence for $\mathrm{H} 2 \mathrm{O} 2$ as an intermediate. J Biol Chem. 1991;266(4):2112-4.

12. Takasu N, Komiya I, Asawa T, Nagasawa Y, Yamada T. Streptozocinand alloxan-induced $\mathrm{H} 2 \mathrm{O} 2$ generation and DNA fragmentation in pancreatic islets. $\mathrm{H} 2 \mathrm{O} 2$ as mediator for DNA fragmentation. Diabetes. 1991;40(9):1141-5.

13. Ahmad FK, He Z, King GL. Molecular targets of diabetic cardiovas cular complications. Curr Drug Targets. 2005;6(4):487-94.

14. Akhani SP, Vishwakarma SL, Goyal RK. Anti-diabetic activity of Zingiber officinale in streptozotocin-induced type I diabetic rats. JPharm Pharmacol. 2004;56(1):101-5.

15. Chen V, Ianuzzo CD. Dosage effect of streptozotocin on rat tissue enzyme activities and glycogen concentration. Can J Physiol Pharmacol. 1982;60(10):1251-6.

16. Huang $\mathrm{CN}$, Horng JS, Yin MC. Antioxidative and antiglycative effects of six organosulfur compounds in low-density lipoprotein and plasma.J Agric Food Chem. 2004;52(11):3674-8.

17. S. Sharma S, K. Gupta Y. Reversal of cisplatin-induced delay in gastric emptying in rats by ginger (Zingiber officinale). J Ethnopharmacol.1998;62(1):49-55.

18. Young HY, Luo YL, Cheng HY, Hsieh WC, Liao JC, Peng WH. Analgesic and anti-inflammatory activities of [6]-gingerol. J Ethnophar macol. 2005;96(1-2):207-10.

19. Katiyar SK, Agarwal R, Mukhtar H. Inhibition of tumor promotion in SENCAR mouse skin by ethanol extract of Zingiber officinale rhizome. Cancer Res. 1996;56(5):1023-30.

20. Li WG, Zhang XY, Wu YJ, Tian X. Anti-inflammatory effect and mechanism of proanthocyanidins from grape seeds. Acta Pharmacol Sin. 2001;22(12):1117-20.

21. Zancan Kelly C, Marques Marcia OM, Petenate Ademir J, Meireles MAngela A. Extraction of ginger (Zingiber officinale Roscoe) oleoresin with $\mathrm{CO} 2$ and co-solvents: a study of the antioxidant action of the extracts. J Supercrit Fluids. 2002;24(1):57-76.

22. Shanmugam KR, Mallikarjuna K, Kesireddy N, Sathyavelu Reddy $K$. Neuroprotective effect of ginger on anti-oxidant enzymes in streptozotocin-induced diabetic rats. Food Chem Toxicol 2011;49(4):893-7.

23. Ohkuwa T, Sato Y, Naoi M. Hydroxyl radical formation in diabetic rats induced by streptozotocin. Life Sci. 1995;56(21):1789-98.

24. Halliwell Barry, Kaur Harparkash, Ingelman-Sundberg Magnus. Hydroxylation of salicylate as an assay for hydroxyl radicals: A cautionary note. Free Radical Biol Med.1991;10(6):439-441.

25. Grootveld M, Halliwell B. Aromatic hydroxylation as a potential measure of hydroxyl-radical formation in vivo. Identification of hydroxylated derivatives of salicylate in human body fluids. Biochem J. 1986;237(2):499-504.

26. Bhandari U, Kanojia R, Pillai KK. Effect of ethanolic extract of Zingiber officinale on dyslipidaemia in diabetic rats. JEthnopharmacol. 2005;97(2):227-30

27. Mooradian AD. Antioxidants and diabetes. Nestle Nutr Workshop Ser Clin Perform Programme. 2006;11:107-22.

28. Sheikh-Ali M, Chehade JM, Mooradian AD. The antioxidant paradox in diabetes mellitus. Am J Ther. 2011;18(3):266-78.

29. Maritim AC, Sanders RA, Watkins JB, 3rd. Diabetes, oxidative stress, and antioxidants: a review. J Biochem Mol Toxicol. 2003;17(1):24-38

30. Creager MA, Luscher TF, Cosentino F, Beckman JA. Diabetes and vascular disease: pathophysiology, clinical consequences, and medical therapy: Part I. Circulation. 2003;108(12):1527-32.

31. Bokov A, Chaudhuri A, Richardson A. The role of oxidative damage and stress in aging. Mech Ageing Dev. 2004;125(10-11):811-26.

32. Sen CK. Oxygen toxicity and antioxidants: state of the art. Indian JPhysiol Pharmacol.1995;39(3):177-96.

33. Kavalalı G, Tuncel H, Göksel S, Hatemi HH. Hypoglycemic activity of Urtica pilulifera in streptozotocin-diabetic rats. J Ethnopharmacol. 2003;84(2-3):241-245.

34. Lipinski Boguslaw. Pathophysiology of oxidative stress in diabe 
Ahmadi R et al.

tes mellitus. J Diabet Complications. 2001;15(4):203-210.

35. Obata Toshio, Yamanaka Yasumitsu. Glibenclamide, an antagonist of ATP sensitive $\mathrm{K}+$ channels, blocks free radical generation in the rat myocardium. Neuroscience Letters. 1998;257(1):5759.

36. Rajkumar L, Srinivasan N, Balasubramanian K, Govindarajulu
P. Increased degradation of dermal collagen in diabetic rats. Indian J Exp Biol. 1991;29(11):1081-3.

37. Huie,R.E , Neta,P. , D.L.Gilbert CAColton. Chemistry of reactive oxygenspecies: reactive oxygen species in biological systems. Hure,R.E, Neta,P. , D.L.Gilbert CAColton, editors. NewYork: Kluwer Academic; 1999. 